\title{
Association of Serum HE4 with Primary Tumor Diameter and Depth of Myometrial Invasion in Endometrial Cancer Patients at Rajavithi Hospital
}

\author{
Nisa Prueksaritanond $^{1,2 *}$, Patchara Cheanpracha ${ }^{1}$, Marut Yanaranop ${ }^{1,2}$
}

\begin{abstract}
Background: Although there are no biomarkers that are routinely used in endometrial cancer (EC) management, many studies have found that serum human epididymis protein 4 (HE4) is superior to cancer antigen $125(\mathrm{CA125})$ in the detection of EC. The correlation of HE4 with two prognostic factors for EC, primary tumor diameter (PTD) and depth of myometrial invasion (DMI) may be useful in identifying EC patients at high risk of lymphatic dissemination. Objective: To evaluate the correlation of serum HE4 with PTD and DMI in patients with EC. Materials and Methods: A cross-sectional study was conducted on 70 EC patients who were scheduled for elective surgery at Rajavithi Hospital between 1st September 2013 and 30th May 2014. Preoperative serum levels of $\mathrm{HE} 4$ and $\mathrm{CA1} 25$ were investigated, and then gross measurement of PTD was taken and postoperative pathologic slides were reviewed for DMI including histologic types, grading and staging. Results: Preoperative serum HE4 levels were strongly correlated with PTD $(r=0.65, p<0.001)$ and moderately correlated with DMI $(r=0.46, p<0.001)$. Moreover, serum HE4 levels were significantly elevated in EC patients with PTD $>2 \mathrm{~cm}(\mathrm{p}<0.001)$ and DMI $>50 \%(\mathrm{p}=0.004)$. The performance of serum HE4 in identifying EC patients at low risk and high risk of lymph node metastasis was significantly better than that of CA125 (AUC 0.88 vs. $0.65, \mathrm{p}=0.003$ ). At an optimal cut-off value of $70 \mathrm{pM} / \mathrm{L}$, serum $\mathrm{HE} 4$ had a sensitivity of $83.3 \%$ and a specificity of $80.0 \%$. Conclusions: In EC patients, preoperative serum HE4 is significantly correlated with PTD and DMI. Serum HE4 levels could be useful in identifying endometrial cancer patients at high risk of lymphatic spread who would benefit from systemic lymphadenectomy at the cut-off value of $70 \mathrm{pM} / \mathrm{L}$.
\end{abstract}

Keywords: Endometrial cancer - human epididymis protein 4 - CA125 - primary tumor diameter - myometrial invasion

Asian Pac J Cancer Prev, 17 (3), 1489-1492

\section{Introduction}

Endometrial cancer (EC) is the second most common gynecological malignancy worldwide (Jemal et al., 2008) and the third most common after cervical and ovarian cancer in Thailand (Cancer of Thailand, 2010). Mostly, EC patients are diagnosed at an early stage with good prognosis (Creasman et al., 2006; Trovik et al., 2012). Two important prognostic factors for lymph node metastasis and survival of EC patients are primary tumor diameter (PTD) and depth of myometrial invasion (DMI) (Mariani et al., 2000).

According to Mayo Clinic criteria, EC patients at risk of lymphatic dissemination requiring systematic lymphadenectomy (Mariani et al., 2008) can be stratified into two risk categories: a low-risk group consisting of those with endometrioid type, histologic grade 1 or 2 , PTD less than or equal $2 \mathrm{~cm}$. and DMI less than or equal to $50 \%$, or those with endometrioid type and no DMI; and a high-risk group comprising those who do not meet the above criteria (Mariani et al., 2008). In one populationbased study, the low-risk group was associated with a $1.4 \%$ risk of lymph node metastasis, compared to a $6.4 \%$ risk in patients in the high-risk group $(\mathrm{p}<0.001)$ (Vargas et al., 2014).

Currently, measurement of serum Cancer Antigen 125 (CA125) is recommended in the preoperative evaluation of EC patients, as high levels of CA125 are associated with advanced-stage EC. However, serum CA125 exhibits a low sensitivity and specificity (Powell et al., 2005), and only $10-20 \%$ of stage I EC patients present with elevated CA125 concentration (Powell et al., 2005). Human Epididymis Protein 4 (HE4) is a novel biomarker for ovarian cancer and is overexpressed in patients with serous and endometrioid epithelial ovarian carcinoma (Drapkin et al., 2005) however, recent studies have suggested that serum HE4 is also elevated in EC and has a higher sensitivity than CA125 (Moore et al., 2008; Angioli et al., 2013; Antonsen et al., 2013; Brennan et al., 2014). In addition, Kalogera et al found that elevation of serum 


\section{Nisa Prueksaritanond et al}

HE4 levels correlated with PTD and DMI and suggested the potential utility of HE4 in the preoperative prediction of high-risk EC and the necessity for definitive surgical staging (Kalogera et al., 2012).

The purpose of the present study was to evaluate the correlation of preoperative serum HE4 with PTD and DMI in EC patients and to assess the performance of preoperative serum HE4 in distinguishing between lowand high-risk EC.

\section{Materials and Methods}

The present study was a cross-sectional design and was approved by the Institutional Review Board (IRB) of Rajavithi Hospital. EC patients were prospectively enrolled if they had been scheduled for elective surgery at Rajavithi Hospital between September 2013 and May 2014. All participants gave written informed consent. EC patients were excluded if they had been histologically diagnosed with carcinosarcoma, had a history of other cancers, had undergone surgery in other hospitals or had incomplete data.

In the preoperative period, clinical data were collected and $5 \mathrm{ml}$. samples of blood for serum HE4 and CA125 were obtained using peripheral venous puncture 48 hours prior to surgery and processed immediately or stored at $-20^{\circ} \mathrm{C}$ until needed. Clotted blood tubes were centrifuged at $800 \mathrm{~g}$ for 10 minutes, after which the serum was separated. Serum biomarker concentrations were investigated using Cobas ${ }^{\circledR} 6000$ analyzer with Elecsys $\AA$ CA 125 II and Elecsys ${ }^{\circledR}$ HE4 reagent kits.

In the postoperative period, hysterectomy specimens were sent to the cutting room in the Department of Pathology for systematic gross examination and measurement of PTD by a pathologic technician. PTD was taken as the greatest dimension of gross tumor. Systematic examination of uterine specimen for pathologic slides was performed in accordance with the protocol of the College of American Pathologists (CAP) (Movahedi-Lankarani et al., 2009). Pathologic slides were reviewed by Yanaranop M. for histologic types, grading, DMI and staging. DMI was the percentage of depth of myometrial invasion measured from the endometrial junction to the deepest point of invasion over the total myometrial thickness (Ali et al., 2007).

Sample size determination was based on the formula for estimating the correlation coefficient using type I error at 0.05 with a power of $80 \%$. Sample size was calculated with the correlation coefficient used in the study of Kalogera et al. (2012), and a total of at least 70 subjects were required.

Statistical analysis was performed using STATA version 10 (StataCorp, College Station, TX). The baseline characteristics of the participants were described using frequency and percentage for categorical data, and mean, standard deviation, median and range for continuous data. Comparison between the continuous variables was calculated by Student t-test and associations between the continuous variables were established using Pearson's correlation. Assessment of risk groups for lymphatic dissemination was performed using the Mayo Clinic criteria as the classification reference, and ROC curves were plotted with area under the curve (AUC) and its 95\% confidence interval (CI). The optimal cut-off value was identified with the Youden Index method (Youden WJ, 1950). Sensitivity (Sn), specificity (Sp), positive predictive value (PPV) and negative predictive value (NPV) were calculated for diagnostic performance.

\section{Results}

During the study period, a total of $77 \mathrm{EC}$ patients were prospectively enrolled, and 7 patients were excluded: five cases of carcinosarcoma and two cases with incomplete data (no serum HE4 results). Ultimately, 70 EC patients were included for analysis.

Table 1. Baseline Clinicopathologic Characteristics

\begin{tabular}{|c|c|c|}
\hline Characteristics & & $\mathrm{N}=70$ \\
\hline Age (yr), mean (SD) & 56.9 & 10.6 \\
\hline BMI $\left(\mathrm{kg} / \mathrm{m}^{2}\right)$, mean $(\mathrm{SD})$ & 29.8 & 6.5 \\
\hline HE4 (pM/L), median (range) & 133 & $(35.8-956.2)$ \\
\hline CA125 (U/ml), median (range) & 21.6 & $(6.6-506.5)$ \\
\hline $\begin{array}{l}\text { Primary tumor diameter }(\mathrm{cm}) \text {, mean } \\
(\mathrm{SD})\end{array}$ & 4.8 & 2 \\
\hline$\leq 2 \mathrm{~cm}$ & 11 & $15.70 \%$ \\
\hline$>2 \mathrm{~cm}$ & 59 & $84.30 \%$ \\
\hline $\begin{array}{l}\text { Depth of myometrial invasion }(\%) \text {, } \\
\text { mean (SD) }\end{array}$ & 48.4 & 31.5 \\
\hline$\leq 50 \%$ & 41 & $58.60 \%$ \\
\hline$>50 \%$ & 29 & $41.40 \%$ \\
\hline \multicolumn{3}{|l|}{ Histologic grading (\%) } \\
\hline Grade 1 & 26 & $37.10 \%$ \\
\hline Grade 2 & 27 & $38.60 \%$ \\
\hline Grade 3 & 17 & $24.30 \%$ \\
\hline \multicolumn{3}{|l|}{ FIGO staging $2009(\%)$} \\
\hline Stage I & 49 & $70.00 \%$ \\
\hline Stage II & 9 & $12.90 \%$ \\
\hline Stage III & 10 & $14.30 \%$ \\
\hline Stage IV & 2 & $2.90 \%$ \\
\hline \multicolumn{3}{|l|}{ Risk groups } \\
\hline Low risk & 10 & $14.30 \%$ \\
\hline High risk & 60 & $85.70 \%$ \\
\hline
\end{tabular}

$\mathrm{SD}=$ Standard deviation, BMI = Body mass index, HE4 = Human Epididymis Protein 4, CA125 = Cancer Antigen 125, FIGO $=$ The International Federation of Gynecology and Obstetrics

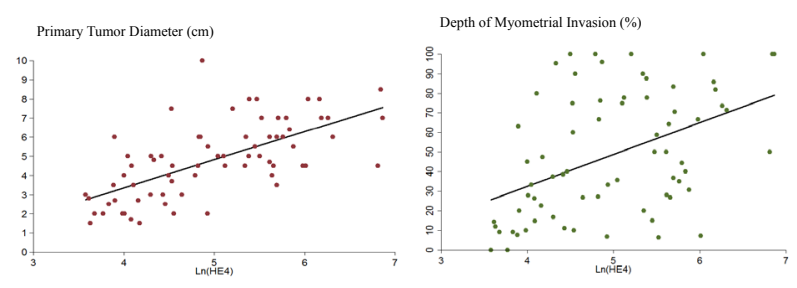

Figure 1. Correlation Between Serum HE4 Level with Primary Tumor Diameter and Depth of Myometrial

Invasion. Abbreviation: $r=$ Pearson's correlation coefficient, Ln = Natural logarithm, HE4 = Human Epididymis Protein 4, CA125 = Cancer Antigen 125 
Association of Serum HE4 with Primary Tumor Diameter and Depth of Myometrial Invasion in Endometrial Cancer

Table 2. Comparison of Serum HE4 and CA 125 Levels with Primary Tumor Diameter and Depth of Myometrial Invasion

\begin{tabular}{lcccccc}
\hline Tumor marker & \multicolumn{2}{c}{ PTD } & p-value & & DMI & p-value \\
\hline & $\leq 2 \mathrm{~cm}$ & $>2 \mathrm{~cm}$ & & $\leq 50 \%$ & $>50 \%$ & \\
& $(\mathrm{n}=11)$ & $(\mathrm{n}=59)$ & & $(\mathrm{n}=41)$ & $(\mathrm{n}=29)$ & \\
Ln(HE4), mean (SD) & $4.1(0.4)$ & $5.1(0.8)$ & $<0.001$ & $4.7(0.8)$ & $5.3(0.8)$ & 0.004 \\
Ln(CA 125), mean (SD) & $2.8(0.3)$ & $3.3(0.9)$ & 0.059 & $3.0(0.6)$ & $3.6(1.1)$ & 0.004 \\
\hline
\end{tabular}

Ln = Natural logarithm, HE4 = Human Epididymis Protein 4, CA125 = Cancer Antigen 125, SD = Standard deviation, PTD = Primary tumor diameter, DMI = Depth of myometrial invasion

Table 3. Performance of Serum HE4 and CA125 in Distinguishing between Low- and High-Risk Endometrial Cancer Patients for Lymphatic Dissemination

\begin{tabular}{|c|c|c|c|c|c|c|c|c|}
\hline \multirow[t]{2}{*}{ Tests } & \multicolumn{3}{|c|}{ ROC } & \multirow{2}{*}{$\begin{array}{c}\text { Optimal } \\
\text { cutoff value }\end{array}$} & \multirow[t]{2}{*}{$\operatorname{Sn}(\%)$} & \multirow[t]{2}{*}{$\mathrm{Sp}(\%)$} & \multirow[t]{2}{*}{ PPV (\%) } & \multirow[t]{2}{*}{ NPV(\%) } \\
\hline & $\overline{\mathrm{AUC}}$ & $95 \% \mathrm{CI}$ & $\mathrm{p}$-value & & & & & \\
\hline HE4 & 0.88 & $(0.78-0.98)$ & 0.003 & 70 & 83.3 & 80 & 96.2 & 44.4 \\
\hline CA125 & 0.65 & $(0.51-0.78)$ & Ref. & 23 & 53.3 & 80 & 94.1 & 22.2 \\
\hline
\end{tabular}

HE4 = Human Epididymis Protein 4, CA125 = Cancer Antigen 125, ROC curve = Receiver operating characteristic curve, AUC $=$ Area under the curve, $\mathrm{CI}=$ Confidence interval, $\mathrm{Sn}=$ Sensitivity, $\mathrm{Sp}=$ Specificity, predictive value $\mathrm{PPV}=$ Positive predictive value, $\mathrm{NPV}=\mathrm{Negative}$

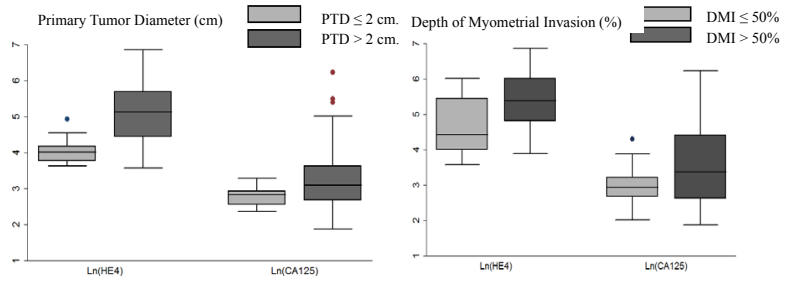

Figure 2. Comparison of Serum HE4 and CA 125 Level with Primary Tumor Diameter and Depth of Myometrial Invasion. Abbreviation: $\mathrm{Ln}=$ Natural logarithm, HE4 = Human Epididymis Protein 4, CA125 $=$ Cancer Antigen 125, PTD = Primary tumor diameter, $\mathrm{DMI}=$ Depth of myometrial invasion

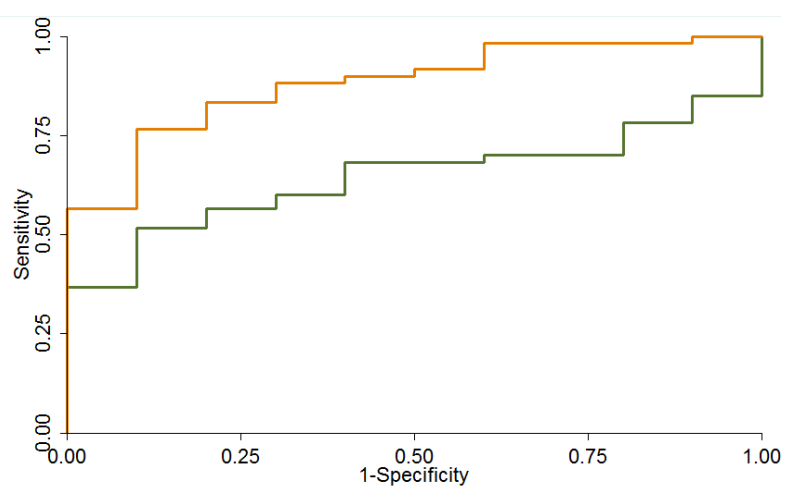

Figure 3. Receiver operating characteristic curve of Serum HE4 and CA 125 Levels for Distinguishing between low- and High-risk Endometrial Cancer. Abbreviation: HE4 = Human Epididymis Protein 4, CA125 = Cancer Antigen 125

Baseline clinicopathologic characteristics of participants are summarized in Table 1 . The mean age was 56.9 years (SD 10.6) and mean body mass index was $29.8 \mathrm{~kg} / \mathrm{m} 2$ (SD 6.5). The median HE4 level was 133 $\mathrm{pM} / \mathrm{L}$ (range 35.8-956.2), and median CA125 level was 21.6 U/ml (range 6.6-506.5). Mean PTD was $4.8 \mathrm{~cm}$ (SD 2.0 ), and $84.5 \%$ of EC patients were found to have PTD of more than $2 \mathrm{~cm}$. Mean DMI was $48.4 \%$ (SD 31.5) and $41.4 \%$ of EC patients revealed myometrial invasion of more than half. The most common histologic grading was grade $2(38.6 \%)$, and most of the patients were stage I EC $(70 \%)$. Patients were divided into two risk groups for nodal metastasis with the low-risk group accounting for $14.3 \%$ and the high-risk group $85.7 \%$.

Correlation between preoperative serum HE4 level and PTD and DMI is shown in Figure 1. Preoperative serum HE4 levels were strongly correlated with PTD ( $r=0.65$, $\mathrm{p}<0.001)$ and moderately correlated with DMI $(\mathrm{r}=0.46$, $\mathrm{p}<0.001)$. Moreover, these serum biomarkers were significantly higher in EC patients with PTD of larger than $2 \mathrm{~cm} .(\mathrm{p}<0.001)$ and DMI of more than $50 \%(\mathrm{p}=0.004)$ as shown in Table 2 and Figure 2. However, preoperative levels of serum CA125 were significantly raised only in EC patients with DMI of more than $50 \%$ ( $\mathrm{p}=0.004)$.

Figure 3 shows a comparison of ROC curve between preoperative serum HE4 and CA125 regarding differentiating EC patients at low risk of lymph node metastasis from those at high risk. The area under ROC curve of serum HE4 was significantly higher than serum CA125 (AUC $=0.88$ vs. 0.65, $\mathrm{p}=0.003$ ). Additionally, the optimal cut-off level of serum HE4 at $70 \mathrm{pM} / \mathrm{L}$ provided a sensitivity of $83.8 \%$ and a specificity of $80 \%$ in differentiating low- and high-risk EC patients.

\section{Discussion}

Nowadays, serum CA125 is the tumor marker that is most commonly used in the management of EC. A prior study has suggested that elevated serum CA125 is correlated with advanced-stage EC (Jhang et al., 2003; Powell et al., 2005) however, another study found that only $10 \%$ of patients with stages I and II EC had elevated serum CA125 (Takeshima et al., 1994).

Several previous studies reported that serum HE4 levels were also elevated in EC (Moore et al., 2008; Bignotti et al., 2011; Kalogera et al., 2012; MutzDehbalaie et al., 2012; Angioli et al., 2013). Kalogera et al. (2012) showed a significant correlation between serum HE4 levels and PTD and DMI (Kalogera et al., 2012). This study confirmed these findings and demonstrated 
that serum HE4 levels strongly correlated with PTD $(\mathrm{r}=0.65, \mathrm{p}<0.001)$ and moderately correlated with DMI $(\mathrm{r}=0.46, \mathrm{p}<0.001)$. Moreover, serum HE4 levels were significantly elevated in patients with PTD of larger than $2 \mathrm{~cm} .(\mathrm{p}<0.001)$ and DMI of more than $50 \%(\mathrm{p}=0.004)$, and this concurs with the findings of previous studies (Moore et al., 2011; Kalogera et al., 2012).

With regard to the risk of nodal metastasis, EC patients in this study were divided into low- and high-risk groups. Angioli R. et al reported that a cut-off point of $70 \mathrm{pM} / \mathrm{L}$ of serum HE4 was the optimal value to detect EC, with a sensitivity of $59.4 \%$ and a specificity of $100 \%$ (Angioli et al., 2013). The present study confirmed this optimal value and found that serum HE4 performed well in differentiating low- from high-risk patients at the cut-off point of $70 \mathrm{pM} / \mathrm{L}$ of serum HE4 (AUC $=0.88, \mathrm{p}=0.003$ ), with a sensitivity of $83.3 \%$ and a specificity of $80 \%$. Hence, this study confirmed that serum HE4 could be used for preoperative risk stratification of EC patients. However, a single parameter of serum HE4 may not be appropriate for this prediction due to the fact that $20 \%$ of false negatives for high-risk EC patients were due to the omission of systematic lymphadenectomy, and this affected survival outcomes. A predictive model of multiple parameters for stratification of risk groups would be useful in any additional study in the future.

Bolstad et al. (2012) reported that higher serum HE4 levels are associated with age and smoking status as much as with creatinine levels and body mass index. In terms of applying HE4 as a biomarker for EC patients, these new finding are very important. The authors also suggested that other factors, such as age, smoking status, creatinine levels and BMI, may affect HE4 levels and should be taken into consideration in order to obtain more accurate results in EC diagnostic and management strategies.

The measurement bias was diminished by using only one technician in the specimen preparation process, and the pathological slides were reviewed by one pathologist alone. However, this study had a limited sampling population and was not validated with other populations.

Bignotti et al. found that high serum HE4 levels correlated with poor survival outcome in poorly differentiated EC patients (Bignotti et al., 2011). The correlation between serum HE4 levels and survival outcome would be an interesting issue for future studies.

In conclusion, preoperative serum HE4 levels are strongly correlated with primary tumor diameter (PTD) and moderately correlated with the depth of myometrial invasion (DMI) in EC. Serum HE4 levels could be used to identify EC patients at high risk of lymphatic dissemination who would benefit from systemic lymphadenectomy at the cut-off value of $70 \mathrm{pM} / \mathrm{L}$.

\section{References}

Ali A, Black D, Soslow RA (2007). Difficulties in assessing the depth of myometrial invasion in endometrial carcinoma. Int J Gynecol Pathol, 26, 115-23.

Angioli R, Plotti F, Capriglione S, et al (2013). The role of novel biomarker HE4 in endometrial cancer: a case control prospective study. Tumour Biol, 34, 571-6.
Antonsen SL, Hogdall E, Christensen IJ, et al (2013). HE4 and CA125 levels in the preoperative assessment of endometrial cancer patients: a prospective multicenter study (ENDOMET). Acta Obstet Gynecol Scand, 92, 1313-22.

Bignotti E, Ragnoli M, Zanotti L, et al (2011). Diagnostic and prognostic impact of serum HE4 detection in endometrial carcinoma patients. Br J Cancer, 104, 1418-25.

Bolstad N, Oijordsbakken M, Nustad K, et al (2012). Human epididymis protein 4 reference limits and natural variation in a Nordic reference population. Tumour Biol, 33, 141-8.

Brennan DJ, Hackethal A, Metcalf AM, et al (2014). Serum HE4 as a prognostic marker in endometrial cancer--a population based study. Gynecol Oncol, 132, 159-65.

Cancer in Thailand (2010). Bangkok, Ministry of public health.

Creasman WT, Odicino F, Maisonneuve P, et al (2006). Carcinoma of the corpus uteri. FIGO 26th Annual Report on the Results of Treatment in Gynecological Cancer. Int $J$ Gynaecol Obstet, 95, 105-43.

Drapkin R, von Horsten HH, Lin Y, et al (2005). Human epididymis protein 4 (HE4) is a secreted glycoprotein that is overexpressed by serous and endometrioid ovarian carcinomas. Cancer Res, 65, 2162-9.

Jemal A, Siegel R, Ward E, et al (2008). Cancer statistics. $C A$ Cancer J Clin, 58, 71-96.

Jhang H, Chuang L, Visintainer P, et al (2003). CA 125 levels in the preoperative assessment of advanced-stage uterine cancer. Am J Obstet Gynecol, 188, 1195-7.

Kalogera E, Scholler N, Powless C, et al (2012). Correlation of serum HE4 with tumor size and myometrial invasion in endometrial cancer. Gynecol Oncol, 124, 270-5.

Mariani A, Dowdy SC, Cliby WA, et al (2008). Prospective assessment of lymphatic dissemination in endometrial cancer: a paradigm shift in surgical staging. Gynecol Oncol, 109, 11-8.

Mariani A, Webb MJ, Keeney GL, et al (2000). Low-risk corpus cancer: is lymphadenectomy or radiotherapy necessary?.Am J Obstet Gynecol, 182, 1506-19.

Moore RG, Brown AK, Miller MC, et al (2008). Utility of a novel serum tumor biomarker HE4 in patients with endometrioid adenocarcinoma of the uterus. Gynecol Oncol, 110, 196-201.

Moore RG, Miller CM, Brown AK, et al (2011). Utility of tumor marker HE4 to predict depth of myometrial invasion in endometrioid adenocarcinoma of the uterus. Int J Gynecol Cancer, 21, 1185-90.

Movahedi-Lankarani S, Gilks C, Soslow R, et al (2009). Protocol for the examination of specimens from patients with carcinoma of the endometrium. college of american pathologists (CAP).

Mutz-Dehbalaie I, Egle D, Fessler S, et al (2012). HE4 is an independent prognostic marker in endometrial cancer patients. Gynecol Oncol, 126, 186-91.

Powell JL, Hill KA, Shiro BC, et al (2005). Preoperative serum CA-125 levels in treating endometrial cancer. $J$ Reprod Med, 50, 585-90.

Takeshima N, Shimizu Y, Umezawa S, et al (1994). Combined assay of serum levels of CA125 and CA19-9 in endometrial carcinoma. Gynecol Oncol, 54, 321-6.

Trovik J, Mauland KK, Werner HM, Wik E, et al (2012). Improved survival related to changes in endometrial cancer treatment, a 30-year population based perspective. Gynecol Oncol, 125, 381-7.

Vargas R, Rauh-Hain JA, Clemmer J, et al (2014). Tumor size, depth of invasion, and histologic grade as prognostic factors of lymph node involvement in endometrial cancer: a SEER analysis. Gynecol Oncol, 133, 216-20.

Youden WJ (1950). Index for rating diagnostic tests. Cancer, 3, 32-5. 